Tropical Journal of Pharmaceutical Research September 2017; 16 (9): 2135-2140

ISSN: 1596-5996 (print); 1596-9827 (electronic)

(C) Pharmacotherapy Group, Faculty of Pharmacy, University of Benin, Benin City, 300001 Nigeria.

All rights reserved.

Available online at http://www.tjpr.org

Original Research Article

http://dx.doi.org/10.4314/tjpr.v16i9.13

\title{
Therapeutic effects of Jiaotai pill on rat insomnia via regulation of GABA signal pathway
}

\author{
Na-na Tang ${ }^{1,2}$, Chang-wen $\mathrm{Wu}^{1}$, Ming-qi Chen ${ }^{3}$, Xue-ai Zeng ${ }^{3}$, Xiu-feng Wang ${ }^{3}$, \\ Yu Zhang ${ }^{3}$ and Jun-shan Huang ${ }^{1,3 *}$ \\ ${ }^{1}$ Fujian University of Traditional Chinese Medicine, Fuzhou, Fujian, 350122, ${ }^{2}$ Jiangxi University of Traditional Chinese Medicine, \\ Nanchang, Jiangxi, 330004, ${ }^{3}$ The Sleep Research Center of Fujian Provincial Institute of Traditional Chinese Medicine, Fuzhou, \\ Fujian 350003, China.
}

*For correspondence: Email: hjsfjtcm@163.com

Sent for review: 27 January 2017

Revised accepted: 5 August 2017

\begin{abstract}
Purpose: To investigate the therapeutic effects of Jiaotai pill (JTP) on rats with insomnia induced by $p$ chlorophenylalanine (PCPA).

Methods: Rats with PCPA-induced insomnia were divided into 5 groups $(n=10)$, made up of control group, positive treatment group (estazolam $0.1 \mathrm{mg} / \mathrm{kg}$ ), and 3 JTP treatment groups (0.6, 1.2 and 2.4 $\mathrm{g} / \mathrm{kg}$ ). Another group of 10 rats were treated as normal group. Rats in normal and control groups were treated with normal saline $(10 \mathrm{~mL} / \mathrm{kg})$. After 14 days of drug treatment, the rats were injected intraperitoneally with sodium pentobarbital $(45 \mathrm{mg} / \mathrm{kg}$ ) and thereafter, latent period and sleeping time were recorded, while contents of $y$-aminobutyric acid (GABA) and glutamic acid (Glu) in hypothalamus were determined by high performance liquid chromatography (HPLC). Furthermore, the expressions of glutamate decarboxylase 65 (GAD-65), glutamate decarboxylase 67 (GAD-67), GABAaminotransferase (GABA)-T, anti-GABA transporter 1 (GAT)-1, anti-GABA transporter (GAT)-3, and $G A B A$ receptors (GABA-A and $G A B A-B$ ) in the hypothalamus were analyzed by western blotting assay. Results: The results showed that JTP $(0.6,1.2$ and $2.4 \mathrm{~g} / \mathrm{kg})$ significantly shortened latent period and prolonged sleeping time $(p<0.01)$. JTP also increased GABA level $(p<0.01)$, but decreased Glu contents of the rat hypothalamus $(p<0.01)$. Western blotting data indicate that JTP significantly upregulated the levels of GAD-65 ( $p<0.01), G A D-67(p<0.05), G A T-1(p<0.01)$, GAT-3 $(p<0.01)$, GABA-A $(p<0.01)$ and GABA-B $(p<0.01)$, while the level of GABA-T was down-regulated.

Conclusion: The results demonstrate that JTP possesses significant sedative effects on insomnia in rats, most probably through a mechanism involving GABA signal pathway.
\end{abstract}

Keywords: Jiaotai pill, Insomnia, GABA, Glutamate, Estazolam, GABA signal pathway

Tropical Journal of Pharmaceutical Research is indexed by Science Citation Index (SciSearch), Scopus, International Pharmaceutical Abstract, Chemical Abstracts, Embase, Index Copernicus, EBSCO, African Index Medicus, JournalSeek, Journal Citation Reports/Science Edition, Directory of Open Access Journals (DOAJ), African Journal Online, Bioline International, Open-J-Gate and Pharmacy Abstracts

\section{INTRODUCTION}

Insomnia is one of the most common clinical diseases, and is also the most common sleep disorder $[1,2]$. It is reported that the occurrence of insomnia is often related to psychological depression and anxiety, negative emotions caused by life events or disease [3]. Results of epidemiological studies indicate that the incidence of insomnia in adolescents and the elderly are approximately 11 and $40 \%$, respectively $[3,4]$. Thus, research efforts have increasingly focused on the search for insomnia therapy. The anti-insomnia drugs currently in clinical use are mainly western medications, amongst which are benzodiazepine receptor agonists. However these drugs are associated with side-effects, dependence and tolerance over 
time. It has been demonstrated that Chinese traditional medicine system has significant effect on insomnia, and it also has low adverse side effect [5].

Jiaotai pill (JTP) is a traditional Chinese medicinal preparation which contains two Chinese herbal medicines (Coptidis rhizoma and Cinnamomi cortex) [6]. Many studies have shown that JTP has the significant therapeutic effects on insomnia [6-8]. However, the possible mechanism of JTP-induced therapy of insomnia has not been studied clearly so far. The present study was designed to investigate the effects of JTP on insomnia, and to explore the underlying mechanisms.

\section{EXPERIMENTAL}

\section{Chemicals and reagents}

The reference standards ( $\gamma$-aminobutyric acid and glutamic acid), $p$-chlorophenylalanine (PCPA), ortho-phthalaldehyde and sodium pentobarbital were obtained from Sigma-Aldrich (St. Louis, MO, USA). Estazolam was obtained from Kunming Zhenhua Pharmaceutical Co. (Kunming, China). The primary antibodies of glutamate decarboxylase (GAD)-65, GAD-67, yaminobutyric acid transaminase (GABA-T), antiGABA transporter (GAT)-1, anti-GABA transporter (GAT)-3, GABA receptors (GABA-A and GABA-B) were purchased from Abcam Co. (Cambridge, MA, USA). All other chemical reagents used in this study were of analytical grade.

\section{Animals}

Male specific pathogen-free (SPF) rats (weighing 180 - $220 \mathrm{~g}$ ) were purchased from the Laboratory Animal Center of Fujian Medical University (Fuzhou, China). The animals were maintained under controlled conditions with natural lighting, and allowed free access to food and water. All the animal experimental protocols were performed in accordance with "Principles of Laboratory Animal Care" (NIH publication no. 8523, revised 1985) [9], and were approved by the Animal Care and Use Committee of Fujian University of Traditional Chinese Medicine [no. 2016-04-05].

\section{Animal grouping and treatment}

Insomnia animal model was established according to previously reported method with minor modifications [10]. Male rats were administrated PCPA by intraperitoneal injection (350 mg/kg, i.p.) once a day for 2 days. A total 50 male insomnia rats were divided into 5 groups ( $\mathrm{n}$ $=10$ ), which consisted of model group, positive treated group and 3 JTP treated groups (0.6, 1.2 and $2.4 \mathrm{~g} / \mathrm{kg}$ ). In addition, a group of 10 insomnia-free rats served as normal control. Rats in normal and model groups were treated with normal saline $(10 \mathrm{~mL} / \mathrm{kg})$. After 14 days of continuous drug/saline treatment, all the rats were injected i.p. with sodium pentobarbital (45 $\mathrm{mg} / \mathrm{kg}$ ). Then, the sleep latency and sleep time were recorded, and the rats were sacrificed by decollation. The hypothalamus of each rat was carefully excised and stored at $-80{ }^{\circ} \mathrm{C}$ prior to analysis.

\section{Determination of GABA and Glu contents of hypothalamus}

The levels of GABA and Glu in the hypothalamus were determined by HPLC method with precolumn derivatization assays [7,11]. In this procedure, $1 \mathrm{~mL} \mathrm{HClO}{ }_{4}(0.1 \mathrm{~mol} / \mathrm{L})$ was added to $0.2 \mathrm{~g}$ hypothalamic tissue, and the tissue was then homogenized at $4{ }^{\circ} \mathrm{C}$ and centrifuged at $12000 \mathrm{rpm}$ for $20 \mathrm{~min}$ at the same temperature. To $40 \mu \mathrm{L}$ of the supernatant fraction was added $40 \mu \mathrm{L}$ of orthophthalaldehyde (OPA) solution, and mixed. The resultant solution was kept for few minutes in the dark. The OPA solution was prepared by mixing $10 \mathrm{mg}$ OPA, $1 \mathrm{~mL}$ methanol, $9 \mathrm{~mL}$ boric acid buffer $(\mathrm{pH}=11,0.04 \mathrm{~mol} / \mathrm{L})$ and $40 \mu \mathrm{L}$ 2-mercaptoethanol. The solution was filtered through a $0.22 \mu \mathrm{m}$ filter before HPLC analysis. GABA and Glu standards were labeled in the same manner as the hypothalamic tissue samples.

The OPA-labeled hypothalamic tissue samples and standards were analyzed by HPLC (Shimadzu, LC-2010A, Japan) with $\mathrm{C}_{18}$ column (4.6 mm $\times 250 \mathrm{~mm}, 5 \mu \mathrm{m})$. Solution A was phosphate buffer $(25 \mathrm{mmol} / \mathrm{L}, \mathrm{pH} 6.8)$ with $3 \%$ acetonitrile. Solution B was phosphate buffer (25 $\mathrm{mmol} / \mathrm{L}, \mathrm{pH}$ 6.8) with $50 \%$ acetonitrile. The mobile phase was a mixture of solution $A$ and solution $\mathrm{B}$ according to the following gradient: 0 15 min, $15 \%$ solution B; 15 - 50 min, $30 \%$ solution. The flow rate and the temperature of the column were set to $1.0 \mathrm{~mL} / \mathrm{min}$ and $30{ }^{\circ} \mathrm{C}$, respectively. The wavelengths for fluorescence detection and excitation were set at 425 and 338 $\mathrm{nm}$, respectively.

\section{Western blotting assay}

The hypothalamic tissues were cut into small sections and treated with lysis buffer containing protease and phosphatase inhibitors. The supernatant was obtained by centrifugation at 
$12000 \mathrm{rpm}$ for $15 \mathrm{~min}$ at $4{ }^{\circ} \mathrm{C}$. Total protein concentration of the supernatant was measured using BCA protein assay reagent (Pierce, Rockford, IL, US). Then, $35 \mu \mathrm{g}$ proteins were separated by $12 \%$ SDS/PAGE, transferred to polyvinylidene difluoride (PVDF) membrane and probed with various primary antibodies (GAD-65, GAD-67, GABA-T, GAT-1, GAT-3, GABA-A and GABA-B), followed by incubation with the secondary antibodies. The target protein bands were visualized by chemiluminescence detection, and GAPDH was used to normalize the protein loading.

\section{Statistical analysis}

All data are expressed as mean \pm standard deviation (SD) and group comparison was performed by ANOVA (SPSS 16.0). $P<0.05$ was considered statistically significant $\left({ }^{*}\right.$ and ${ }^{* *}$ represent significance at 0.05 and 0.01 levels, respectively).

\section{RESULTS}

\section{Effect of JTP on latent period and sleeping time}

The effects of JTP on sleep latency and sleep time of rats with insomnia are presented in Figures 1 and Figure 2. The results showed that the latent period of model rat was significantly longer than that of normal rat, whereas the sleeping time was remarkably shorter, indicating that the insomnia model was successfully established. The latent periods of rats in the positive treated group and 3 treated JTP groups $(0.6, \quad 1.2$ and $2.4 \mathrm{~g} / \mathrm{kg})$ were significantly decreased when compared with that of model rats $(p<0.01)$. Furthermore, the sleeping time of JTP treated rats was significantly longer than that of model rats $(p<0.01)$.

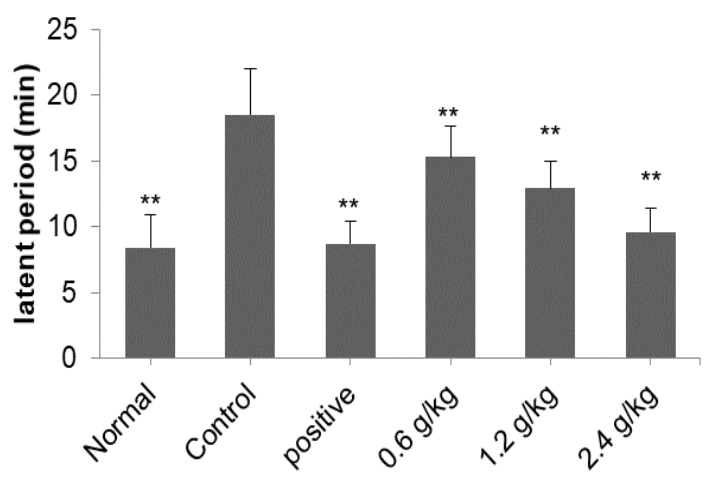

Figure 1: Effect of Jiaotai pill on latent period of insomnia rats. Data are expressed as mean $\pm S D(n=$ 10); ${ }^{*} p<0.05,{ }^{* *} p<0.01$, compared with model group

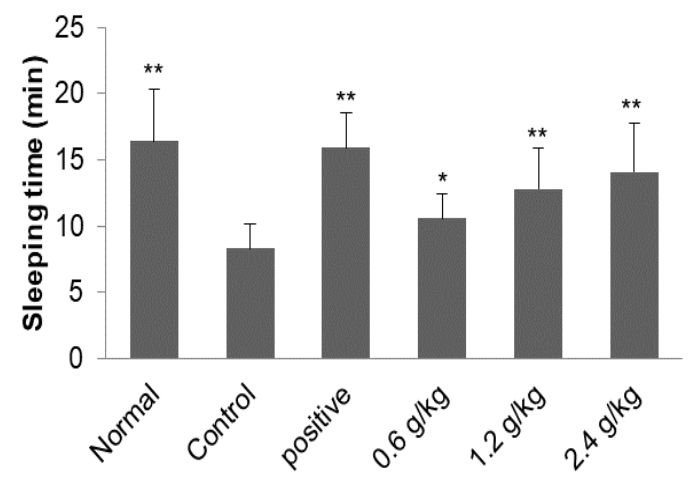

Figure 2: Effect of Jiaotai pill on sleeping time of insomnia rats. Data are expressed as mean \pm SD $(n=$ $10),{ }^{*} p<0.05,{ }^{* *} p<0.01$, compared with model group

\section{Contents of GABA and Glu in hypothalamus}

The levels of GABA and Glu in hypothalamus of the rats are shown in Figure 3 and Figure 4. GABA content of rat in model group was significantly lower than that of normal rat $(p<$ 0.01). However, Glu levels in model rats were significantly increased $(p<0.01)$. More importantly, after treatment with JTP $(0.6,1.2$ and $2.4 \mathrm{~g} / \mathrm{kg}$ ), GABA contents were significantly increased $(p<0.05, p<0.01)$ whereas Glu levels were significantly decreased, when compared with the model rats $(p<0.01)$.

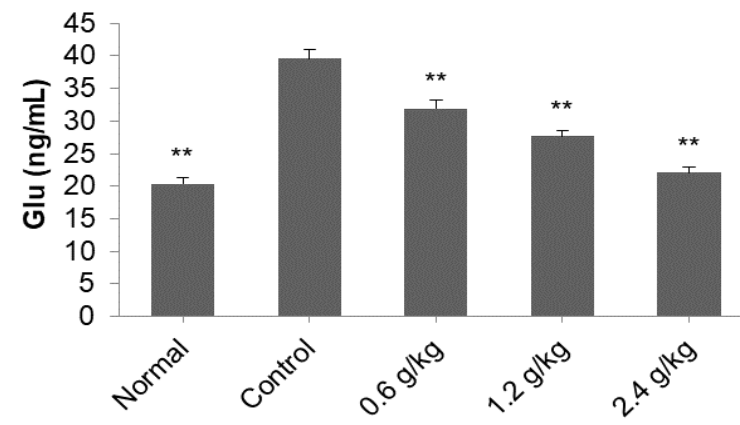

Figure 3: Effect of Jiaotai pill on Glu of insomnia rats. Data are expressed as mean \pm SD $(n=10),{ }^{*} p<0.05$, ${ }^{* *} p<0.01$, compared with model group

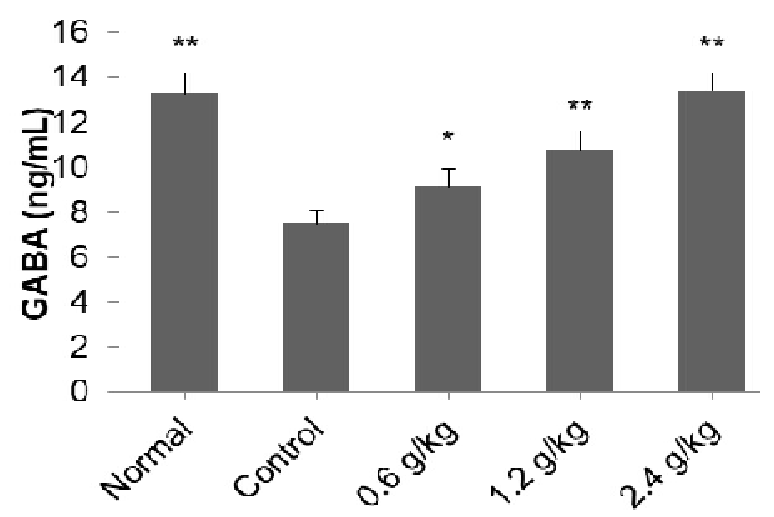

Figure 4: Effect of Jiaotai pill on GABA of insomnia rats. Data are expressed as mean $\pm \mathrm{SD}(\mathrm{n}=10),{ }^{*} p<$ $0.05,{ }^{* *} p<0.01$, compared with model group 


\section{Effect of Jiaotai pill on expressions of GABA signal-related proteins}

As can be seen from Figure 5, expressions of GAD-65, GAD-67, GAT-1, GAT-3, GABA-A and GABA-B were down-regulated in insomnia rats when compared to the normal rats $(p<0.01)$. However, JTP at dose of $2.4 \mathrm{~g} / \mathrm{kg}$ significantly up-regulated expressions of GAD-65 $(p<0.01)$, GAD-67 $(p<0.05)$, GAT-1 $(p<0.01)$, GAT-3 $(p<$ $0.01)$, GABA-A $(p<0.01)$ and GABA-B $(p<0.01)$ in hypothalamus of insomnia rats, when compared with untreated insomnia rats. Furthermore, GABA-T was up-regulated in insomnia rats relative to normal rats $(p<0.01)$, and the JTP $(2.4 \mathrm{~g} / \mathrm{kg})$ treatment down-regulated GABA-T expression in insomnia rats compared to model rats $(p<0.01)$.

\section{DISCUSSION}

GABA and Glu, two major neurotransmitters, exert effects on the excitation/inhibition balance in the central nervous system (CNS) [12]. GABA, an important non-protein amino acid, has inhibitory effects on CNS through blockage of excessive excitement of excitatory neurons [11]. In addition, Glu is recognized as an important excitatory neurotransmitter [13]. Therefore, the contents of GABA and Glu could be used to reflect the degree of insomnia. In the present study, JTP increased the GABA content of hypothalamus of insomnia rats, but decreased the Glu content. This indicates that JTP is a potential source of drug (s) for relieving symptoms of insomnia.
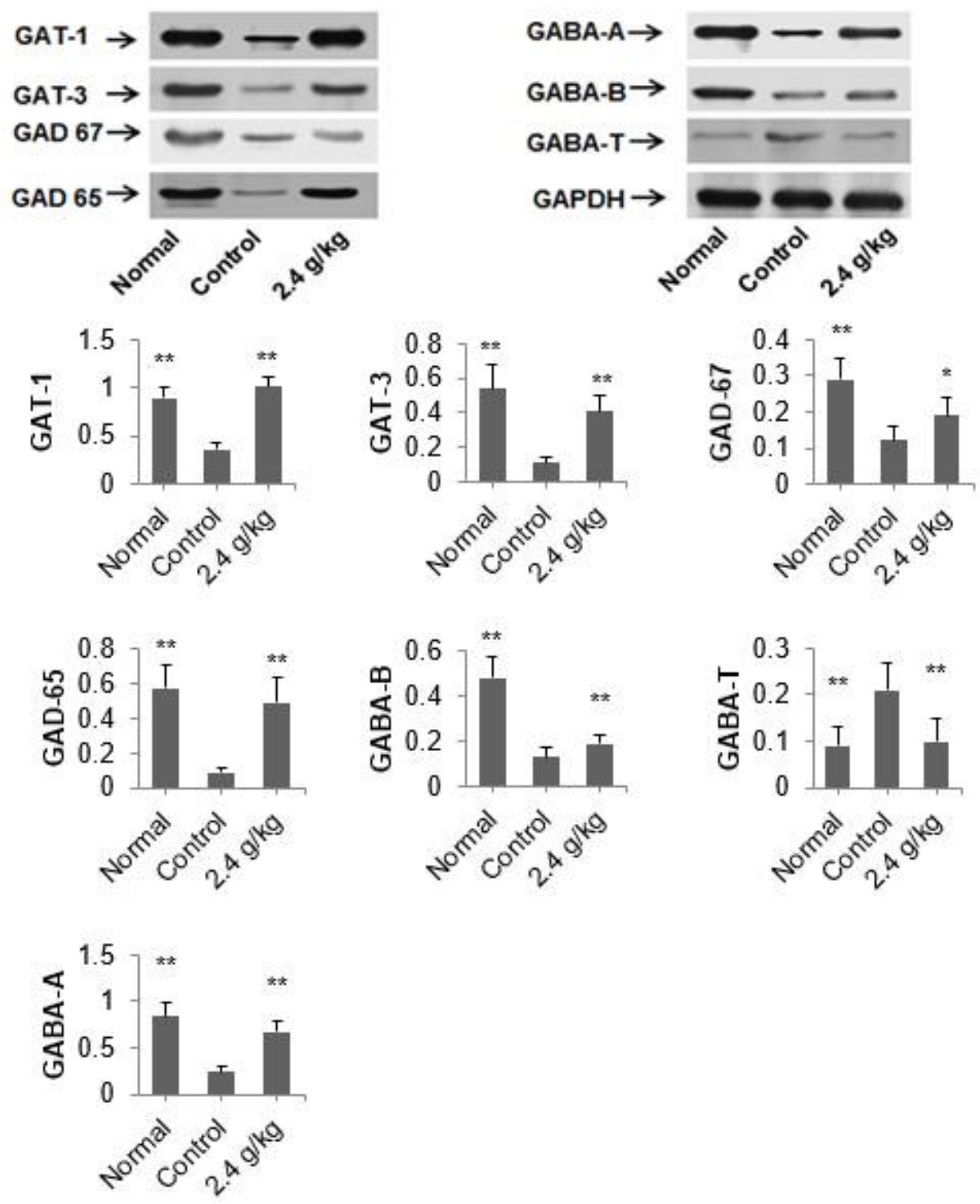

Figure 5: Effect of Jiaotai pill on expressions of GABA signal-related proteins in insomnia rats. 1-3 represented the Normal, Model and JTP $(2.4 \mathrm{~g} / \mathrm{kg})$, respectively. Data were expressed as mean $\pm \mathrm{SD}(\mathrm{n}=10) ;{ }^{*} p<0.05,{ }^{* *} p$ $<0.01$, compared with model group 
The GABA signal pathway performs two functions: synthesis and transport of GABA, and synaptic and non-synaptic release of GABA [14]. The synaptic release of GABA is related to certain proteins such as GAD-65, GAD-67 and GABA-T, which are responsible for converting glutamate to the GABA $[15,16]$. The transport of GABA neurotransmitter is related to GABA transporters such as GAT-1, GAT-1, GAT-1 and GAT-3, which belong to the solute carrier 6 genes family A (SLC6A) of $\mathrm{Na}^{+}$-dependent transporters [17].

GAT regulates the GABA concentration in the brain and is used as new drug for the treatment of treating several CNS diseases [17-19]. In addition, GABA signal pathway is mediated by the two major GABA receptors (GABA-A and GABA-B). GABA-A receptor responds to GABA release by producing excitatory and inhibitory cellular responses. The inhibitory effects of GABA-B on GABA release influences circadian control [14]. In the present study, results of western blotting assay showed that JTP upregulated the expressions of GAD-65, GAD-67, GAT-1, GAT-3, GABA-A and GABA-B, whereas it down-regulated the expression of GABA-T.

\section{CONCLUSION}

The results obtained in the present study demonstrate that JTP exerts sedative effects on PCPA-insomnia in rat. The probable mechanism of action is related to regulation of GABA signal pathway. These findings provide some scientific bases for the clinical use of Jiaotai pill in the treatment of insomnia.

\section{DECLARATIONS}

\section{Acknowledgement}

This work was supported by National Natural Science Foundation of China (no. 81173149).

\section{Conflict of Interest}

No conflict of interest associated with this work.

\section{Contribution of Authors}

The authors declare that this work was done by the authors named in this article and all liabilities pertaining to claims relating to the content of this article will be borne by them.

\section{Open Access}

This is an Open Access article that uses a funding model which does not charge readers or their institutions for access and distributed under the terms of the Creative Commons Attribution License (http://creativecommons.org/licenses/by/ 4.0) and the Budapest Open Access Initiative (http://www.budapestopenaccessinitiative.org/rea d), which permit unrestricted use, distribution, and reproduction in any medium, provided the original work is properly credited.

\section{REFERENCES}

1. Combs D, Goodwin JL, Quan SF, Morgan WJ, Shetty S, Parthasarathy S. Insomnia, Health-Related Quality of Life and Health Outcomes in Children: A Seven Year Longitudinal Cohort. Sci Rep 2016; 6: 27921 - 27921.

2. Kim KW, Kang SH, Yoon IY, Lee SD, Ju G, Han JW, Kim $\mathrm{TH}$, Lee CS, Kim T. Prevalence and clinical characteristics of insomnia and its subtypes in the Korean elderly. Arch Gerontol Geriatr 2017; 68: 68-75.

3. Alvaro PK, Roberts RM, Harris JK, Bruni O. The direction of the relationship between symptoms of insomnia and psychiatric disorders in adolescents. J Affect Disord 2017; 207: 167-174.

4. Chen DY, He SC, Zhou DY. Traditional Chinese Medicine Treatment for Insomnia: A Systematic Review. Chinese Archives Traditional Chinese Med 2016; 34(1): 105-110.

5. Yu YL, Quan SJ. Effects of Jiao-Tai-Long on $Y$ - amino acid and its receptor in the brain of PCPA rats. Lishizhen Med Materia Med Res 2010; 21(6): $1417-$ 1418.

6. Quan SJ, Jiao MM, Hei SY, Qian LL. Effect of Jiaotai Pills on Hypothalamic Orexin $A$ and Gamma-aminobutyric Acid in Sleep Deprivation Rats. J Guangzhou Univ Trad Chin Med 2015; 32(1): 103 - 105.

7. Yue H, Zhou XY, Li CY, Zou ZJ, Wang SM, Liang SW, Gong MJ. Intervention effects of Jiaotai pills on PCPAinduced insomnia in rats. China J Chin Mater Med 2016; 41(18): 3451-3456.

8. National Institute of Health, USA. Public health service policy on humane care and use of laboratory animals; 2002.

9. Gao JR, Ji WB, Jiang $H$, Chen JF. Effects of Extracts from Ziziphi Spinosae Semen and Schisandrae Chinensis Fructus on Amino Acid Neurotransmitter in Rats with Insomnia Induced by PCPA. J Chin Med Mater 2013; 36(10): 1635-1639.

10. Ding GY, Hou YY, Peng JM, Shen YB, Jiang M, Bai G. On-line near-infrared spectroscopy optimizing and monitoring biotransformation process of $\gamma$-aminobutyric acid. J Pharmaceut Biomed 2016; 6: 171 - 178.

11. Kakeda S, Korogi Y, Moriya J, Ohnari N, Sato T, Ueno S, Yanagihara N, Harada M, Matsuda T. Influence of work shift on glutamic acid and gamma-aminobutyric acid (GABA): Evaluation with proton magnetic resonance spectroscopy at 3T. Psychiatry Res 2011; 192(1): 55-59. 
12. Lv H, Xu TL. Advancement on excitatory amino acid transporters. Chin Pharmacol Bulletin 2000; 16(1): 2223.

13. Albers HE, Walton JC, Gamble KL, McNeill JK, Hummer $D L$. The Dynamics of GABA Signaling: Revelations from the Circadian Pacemaker in the Suprachiasmatic Nucleus. Front Neuroendocrinol 2016; http://dx.doi.org/10.1016/j.yfrne.2016.11.003.

14. Kapolowicz MR, Thompson LT. Acute high-intensity noise induces rapid Arc protein expression but fails to rapidly change GAD expression in amygdala and hippocampus of rats: Effects of treatment with $D$ cycloserine. Hear Res 2016; 342: 69-79.

15. Gey L, Gernert M, Löscher W. Continuous bilateral infusion of vigabatrin into the subthalamic nucleus:
Effects on seizure threshold and GABA metabolism in two rat models. Neurobiol Dis 2016; 91:194 - 208.

16. Sałat K, Podkowa A, Malikowska N, Kern F, Pabel J, Wojcieszak E, Kulig K, Wanner KT, Strach B, Wyska E. Novel, highly potent and in vivo active inhibitor of GABA transporter subtype 1 with anticonvulsant, anxiolytic, antidepressant and antinociceptive properties. Neuropharmacology 2017; 113: 331 - 342.

17. Sałat $K, K$ Kulig K. GABA transporters as targets for new drugs. Future Med Chem 2011; 3: 211e222.

18. Wang ZJ, Zhang $X Q$, Cui $X Y$, Cui SY, Yu B, Sheng ZF, Li SJ, Cao Q, Huang YL, Xu YP, Zhang YH. Glucocorticoid receptors in the locus coeruleus mediate sleep disorders caused by repeated corticosterone treatment. Sci Rep 2015; 5: 9442. 